\title{
Outcomes after aortic graft-to-graft anastomosis with an automated circular stapler: A novel approach
}

\author{
Jay J. Idrees, MD, ${ }^{\mathrm{a}, \mathrm{b}}$ Farhang Yazdchi, MD, MS, ${ }^{\mathrm{b}}$ Edward G. Soltesz, MD, MPH, \\ Andrew M. Vekstein, BS, ${ }^{b}$ Christopher Rodriguez, BA, ${ }^{b}$ and Eric E. Roselli, MD ${ }^{\mathrm{a}, \mathrm{b}}$
}

\section{ABSTRACT}

Objective: Patients with complex aortic disease often require multistaged repairs with numerous anastomoses. Manual suturing can be time consuming. To reduce ischemic time, a circular stapling device has been used to facilitate prosthetic graft-to-graft anastomoses. Objectives are to describe this technique and assess outcomes.

Methods: From February 2009 to May 2014, 44 patients underwent complex aortic repair with a circular end-to-end anastomosis (EEA) stapler at Cleveland Clinic. All patients had extensive aneurysms: 17 after ascending dissection repair, 10 chronic type B dissections, and 17 degenerative aneurysms. Stapler was used during total arch repair as an end-to-side anastomosis $(n=36$; including first stage elephant trunk [ET] in 32, frozen ET in 3) and an end-to-end anastomosis during redo thoracoabdominal repair $(n=11)$. Three patients had the stapler used during both stages of repair. Patients underwent early and annual follow-ups with computed tomography analysis.

Results: There were no bleeds, ruptures, or leaks at the stapled site, but 2 patients died. Complications included 7 reoperations not related to the site of stapled anastomosis and 6 tracheostomies, but there was no paralysis or renal failure. Mean circulatory arrest time was $16 \pm 5$ minutes. Mean follow-up was $26 \pm 17$ months and consisted of imaging before discharge, at 3 to 6 months, and at 1 year. Planned reinterventions included 21 second-stage ET completion: Endovascular $(\mathrm{n}=18)$ and open $(\mathrm{n}=3)$. There were 4 late deaths.

Conclusions: Use of an end-to-end anastomotic automated circular stapler is safe, Descending aortic graft-to-graft anastomosis with an
automated circular stapler.

\section{Central Message}

The use of an end-to-end anastomotic stapler is a safe and effective technique for graft-to-graft anastomosis during aortic surgery.

\section{Perspective}

Patients with extensive aortic disease often require multistage repair involving numerous anastomoses. A durable aortic graft-to-graft anastomosis can be safely performed with an end-to-end anastomotic automated stapler during complex aortic reconstruction.

See Editorial Commentary page 1058. effective, and durable in performing graft-to-graft anastomoses during complex thoracic aortic surgery. Further evaluation and refinement of this technique are warranted. (J Thorac Cardiovasc Surg 2016;152:1052-7)

Patients with extensive aortic aneurysms often require multistage repairs that involve numerous anastomoses. ${ }^{1-4}$ Minimizing circulatory arrest time, as well as cardiac, systemic, and spinal ischemic times, during these complex repairs improves outcomes. ${ }^{1}$ Automation of any of these anastomoses could save time.

Stapling devices are currently used routinely in gastrointestinal surgery and have largely replaced the conventional hand-

From the ${ }^{\mathrm{a} A o r t a}$ Center, and the ${ }^{\mathrm{b}}$ Department of Thoracic and Cardiovascular Surgery, Heart and Vascular Institute, Cleveland Clinic, Cleveland, Ohio.

This study was supported in part by John and Judy Chew and the Warden Foundation. Received for publication March 9, 2016; revisions received June 1, 2016; accepted for publication June 8, 2016; available ahead of print July 19, 2016.

Address for reprints: Eric E. Roselli, MD, Department of Thoracic and Cardiovascular Surgery, Cleveland Clinic, 9500 Euclid Ave, Desk J4-1, Cleveland, OH 44195 -

5108 (E-mail: roselle@ccf.org).

$0022-5223 / \$ 36.00$

Copyright (c) 2016 by The American Association for Thoracic Surgery

http://dx.doi.org/10.1016/j.jtcvs.2016.06.039 sewn anastomosis. ${ }^{5-8}$ We have used a circular endovascular end-to-end anastomotic (EEA) stapler to perform prosthetic graft-to-graft anastomosis during both first and second-stage elephant trunk procedures (Figure 1). We describe the use of this technique and review outcomes.

\section{MATERIALS AND METHODS}

Between February 2009 and May 2014, a total of 44 patients underwent complex aortic repair with a circular EEA stapler (Covidien EEA Stapler; Medtronic, Inc, Minneapolis, Minn) used to perform 47 anastomoses (36 end-to-side and 11 end-to-end) at the Cleveland Clinic. Mean age was

Scanning this QR code will take you to a video for the article. 


\section{Abbreviations and Acronyms \\ $\mathrm{EEA}=$ end-to-end anastomosis \\ $\mathrm{CT}=$ computed tomography}

$61 \pm 14$ years. All patients had extensive degenerative thoracoabdominal disease involving the aortic arch, the thoracic descending aorta, or the thoracoabdominal aorta. Seventeen patients had chronic residual dissection after a previous history of emergency acute type A repair, 10 were seen with chronic type B dissection, and 17 had degenerative thoracoabdominal aneurysm. Mean preoperative maximum aortic diameter was $58 \pm 10 \mathrm{~mm}$ (Table 1).

During the index operation, the EEA stapler was used to perform an end-to-side graft-to-graft anastomosis in the aortic arch in 36 patients $(82 \%)$ and an EEA in the descending aorta in $8(18 \%)$. In addition, 3 patients who had first-stage elephant trunk construction done with the EEA stapler went on to have their second-stage performed in an open fashion, again with the EEA stapler used for the distal graft-to-graft anastomosis. These 47 graft-to-graft anastomoses comprise the experience reported here.

The 36 end-to-side anastomoses included first-stage elephant trunk $(\mathrm{n}=32)$, frozen elephant trunk $(\mathrm{n}=3)$, and total aortic arch repair $(\mathrm{n}=1)$. Four of the patients with descending aortic disease had the index operation performed as an elephant trunk completion but did not have the stapler used during their first-stage elephant trunk repair. The other 4 patients with index descending aortic disease had previous open descending repair for graft-to-graft anastomosis. Of the 11 descending EEA stapler anastomoses, 7 were performed as an elephant trunk completion procedure.

Twenty-seven patients underwent 33 concomitant procedures at the time of the index operation, including coronary bypass $(n=9)$, aortic valve replacement $(n=7)$, tricuspid valve repair $(n=1)$, patent foramen ovale closure $(n=1)$, pulmonary trunk repair $(n=1)$, ligation of the left atrial appendage $(n=2)$, and open distal aortic fenestration $(n=12)$. The last procedure was performed at the time of the first-stage elephant trunk and involved resection of the dissection flap in the retrocardiac segment of the descending aorta to facilitate endovascular elephant trunk completion at a later stage. ${ }^{4}$

All patients required multiple anastomoses for additional procedures, such as reimplantation of the aortic arch head vessels and coronary grafts. Altogether, 303 anastomoses were performed in 44 patients (average of 7 anastomoses per patient) during the index operation at which the EEA stapler was used to perform 1 of the graft-to-graft anastomoses.

\section{Operative Technique}

End-to-side aortic arch repair. With the patient under general anesthesia, and after cannulation of the right axillary artery and sternotomy, the patient was placed on cardiopulmonary bypass and cooled to $22^{\circ} \mathrm{C} .{ }^{9}$ During a period of circulatory arrest and selective antegrade brain perfusion, the aortic arch vessels were reconstructed as a unit or independently with single and multibranched Dacron polyester fabric grafts, respectively. Another Dacron polyester fabric tube graft was then inverted on itself and inserted into the distal arch. It was then sutured in position as a modified elephant trunk. ${ }^{2}$ The aortic elephant trunk graft was then reduced back into the mediastinum. At this point, the EEA stapler (Figure 1) was used to connect the aortic arch vessel graft to the main aortic elephant trunk graft in an end-to-side fashion (Figure 2 and Video 1).

Depending on the diameter of the aortic arch vessel graft, either 21- or 25 -mm $\times 2.5$-mm EEA stapler was selected. The anvil was quickly secured in the aortic arch vessel graft with a polypropylene purse-string suture.
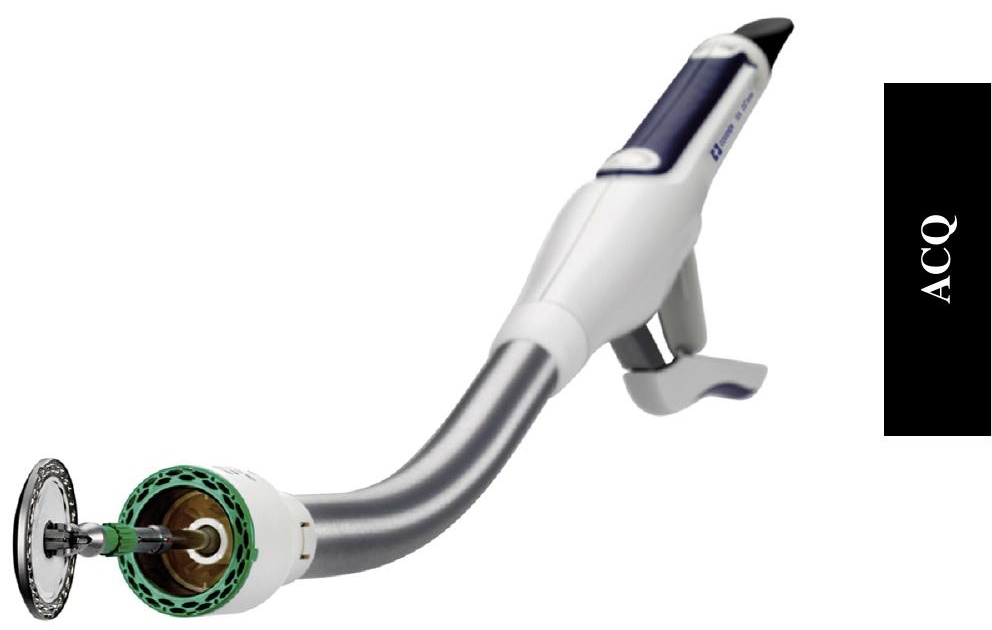

FIGURE 1. End-to-end anastomotic stapler device.

Next, the cartridge-carrying instrument was introduced from proximal end of the main aortic graft and the center rod passed through a small stab incision. The center rod was fitted into the anvil shaft, the instrument was tightened, and the stapler was deployed (Figure 2, $A$ ). Firing the stapler placed a double staggered circle of titanium staples between the 2 grafts and cut a circular orifice to supply the aortic arch vessel graft (Figure 2, $C$ ). The instrument containing the graft "doughnuts" was removed, and the anastomosis was visually inspected. The aortic arch vessels were then deaired, the graft was crossclamped proximal to the EEA stapler anastomosis, and full-flow perfusion and rewarming were resumed. A few sutures were typically placed to ensure graft-to-graft hemostasis after perfusion was resumed. Proximal aortic reconstruction was then completed during the rewarming phase.

End-to-end descending aortic repair. With the patient under general anesthesia and with a double-lumen endotracheal tube and a spinal drainage catheter in place, a thoracoabdominal incision was made through the sixth intercostal space. The diaphragm was incised radially, and the retroperitoneum was dissected to obtain sufficient exposure of the thoracoabdominal aorta, with the extent depending on the pathology. The left common femoral artery and the left inferior pulmonary vein were cannulated for atriofemoral partial bypass and active cooling. After clamping of the distal descending aorta, the previous proximal descending graft was clamped or the proximal descending aorta was opened and the elephant trunk graft was quickly grasped and clamped. ${ }^{2}$ An appropriate multilimb graft was brought to the field. Depending on the diameter of these grafts, either a $21-$ or $25-\mathrm{mm} \times 3.5$-mm EEA stapler was selected. The anvil was tied into the distal end of the previously placed elephant trunk graft with a polypropylene purse-string suture. The cartridge-carrying instrument was introduced into the multilimb graft, and a polypropylene purse-string suture was tightened down around the center rod. The center rod was then mated into the anvil shaft, the instrument was tightened, and the stapler was deployed. This created an end-to-end, graft-to-graft anastomosis (Figure 3). After reimplantation of the intercostal arteries, the aortic clamp was repositioned. A few sutures were sometimes needed to secure graft-to-graft hemostasis. The distal anastomosis was created in the standard fashion, either as separate grafts to each of the visceral arteries (preferred technique) or with a Carrel patch to the visceral vessels.

\section{Follow-up and Imaging}

All patients with aortic disease were followed up with routine computed tomographic (CT) imaging surveillance at discharge, within the first 3 months, at 12 months after surgery, and then annually thereafter. 
TABLE 1. Patient characteristics, operative details, and complications

\begin{tabular}{lc}
\hline \multicolumn{1}{c}{ Total patients } & $\mathbf{4 4}$ \\
\hline Age (y, mean \pm SD) & $61 \pm 14$ \\
Male & $18(75 \%)$ \\
Previous cardiac surgery & $25(57 \%)$ \\
$\quad$ Acute type A dissection repair & $17(39 \%)$ \\
Aortic morphology & \\
Aneurysm & $17(39 \%)$ \\
Type B dissection with aneurysm & $10(23 \%)$ \\
Chronic residual dissection after acute type A repair & $17(39 \%)$ \\
Preoperative mean maximum descending diameter & $58 \pm 10$ \\
$\quad$ (mm, mean \pm SD) & \\
Operative details & \\
Index operation & \\
$\quad$ First-stage elephant trunk completion & $32(73 \%)$ \\
$\quad$ Frozen elephant trunk & $3(7 \%)$ \\
$\quad$ Open second-stage thoracoabdominal completion & $8(18 \%)$ \\
$\quad$ repair & \\
$\quad$ Total aortic arch replacement & $1(2 \%)$ \\
Reintervention & \\
$\quad$ Second-stage elephant trunk completion & $21(48 \%)$ \\
$\quad$ Endovascular & $18(41 \%)$ \\
$\quad$ Open & $3(7 \%)$ \\
TEVAR extension* & $1(2 \%)$ \\
Anastomoses with EEA (n = 47) & \\
End-to-side anastomosis & $36(76 \%)$ \\
EEA & $11(24 \%)$ \\
Complications & \\
Median follow-up (mo, mean \pm SD) & $26 \pm 17$ \\
EEA staple anastomosis leak, rupture, or stricture & $2(4.5 \%)$ \\
Stroke & $0(0 \%)$ \\
Tracheostomy & $1(2 \%)$ \\
Reoperation for chest bleeding & $6(14 \%)$ \\
\hline
\end{tabular}

Data are number with percentage except as noted. $S D$, Standard deviation; TEVAR, thoracic endovascular aortic repair; EEA, end-to-end anastomosis. *One patient had 2 reinterventions that included endovascular elephant trunk completion and a subsequent thoracic endovascular aortic repair extension.

Postoperative CT scans were performed with a standardized contrastenhanced protocol. All these images were analyzed with 3-dimensional reconstruction software (Aquarius; TeraRecon, Inc, Foster City, Calif) to assess for patency of the repair, integrity of the anastomoses, and morphologic characteristics of the aneurysm and dissection (Figure 1, $B$ and $D$ ). Among the 42 patients who survived, mean follow-up for the study was $35 \pm 24$ months (median, 41 months). Postoperative CT imaging was available for $88 \%$ of patients.

\section{Outcomes and Statistics}

Renal failure was defined as the need for hemodialysis, and respiratory failure was defined as the need for postoperative reintubation or tracheostomy. As the total number of events were small, a descriptive analysis was performed. Categoric variables are summarized as frequencies and percentages, and continuous variables are summarized as median or mean $\pm \mathrm{SD}$. Long-term survival estimates were calculated with the Kaplan Meier method. Institutional review board approval with patient consent waived was obtained for the performance of this retrospective study.

\section{RESULTS}

No bleeding, stricture, rupture, leakage, or pseudoaneurysm was observed from the EEA stapler anastomotic suture line either early or at a mean follow-up of $26 \pm 17$ months. There were 2 in-hospital deaths. One patient died of embolic stroke involving the posterior cerebral circulation on postoperative day 2 . This patient previously had severe atherosclerotic disease of the ascending aorta and aortic arch, with a history of permanent stroke and residual arm weakness. The second patient had extensive thoracoabdominal disease and initially underwent first-stage elephant trunk repair with the EEA stapler, with a prolonged initial recovery as a result of respiratory failure. In this case, a second stage had been planned; however, the patient died of descending aortic rupture on postoperative day 34 before the second-stage repair could be performed. Postoperative CT confirmed that the site of the EEA stapler anastomosis was intact. Seven patients underwent reoperation for chest bleeding that was determined by direct inspection not to be related to the site of the EEA stapler anastomosis. Six required tracheostomy for respiratory failure. There were no cases of postoperative paralysis or renal failure. Mean circulatory arrest time was $16 \pm 5$ minutes. Of the 32 patients who were treated with the first-stage elephant trunk as the index operation, $21(66 \%)$ underwent second-stage completion with either an endovascular $(n=18)$ or an open thoracoabdominal $(\mathrm{n}=3)$ approach. Eleven patients did not have completion of the second stage. As mentioned previously, 1 died in the hospital from descending rupture while recovering from the first stage. The remaining $10 \mathrm{pa}-$ tients underwent first-stage elephant trunk as a prophylactic measure. Of these, 4 died of late noncardiac causes and the other 6 are still being followed up with maintenance imaging. Survivals at $1,3,5$, and 8 years were $95 \%, 90 \%, 81 \%$, and $81 \%$, respectively (Figure 4)

\section{DISCUSSION}

The use of a commercially available EEA stapler to perform graft-to-graft anastomosis is feasible and safe. This method allows an efficient and rapid technique for performing large-caliber graft-to-graft anastomoses during complex aortic procedures in which minimizing ischemic time is crucial to surgical success.

Mechanical stapling devices are widely used in gastrointestinal and general thoracic surgery but only have been sparsely used for vascular anastomoses..$^{5-7}$ Some coronary bypass anastomotic devices have been commercially available. ${ }^{10,11}$ Takata and colleagues ${ }^{11}$ recently tested a computer-controlled circular stapler device (SurgAssist iDrive; Power Medical Interventions, Dublin, Ireland) that performs anastomosis of the aorta and an artificial graft. Similarly, Lachat and colleagues ${ }^{12}$ have used covered stent devices bridged across the target vessel and graft to perform 

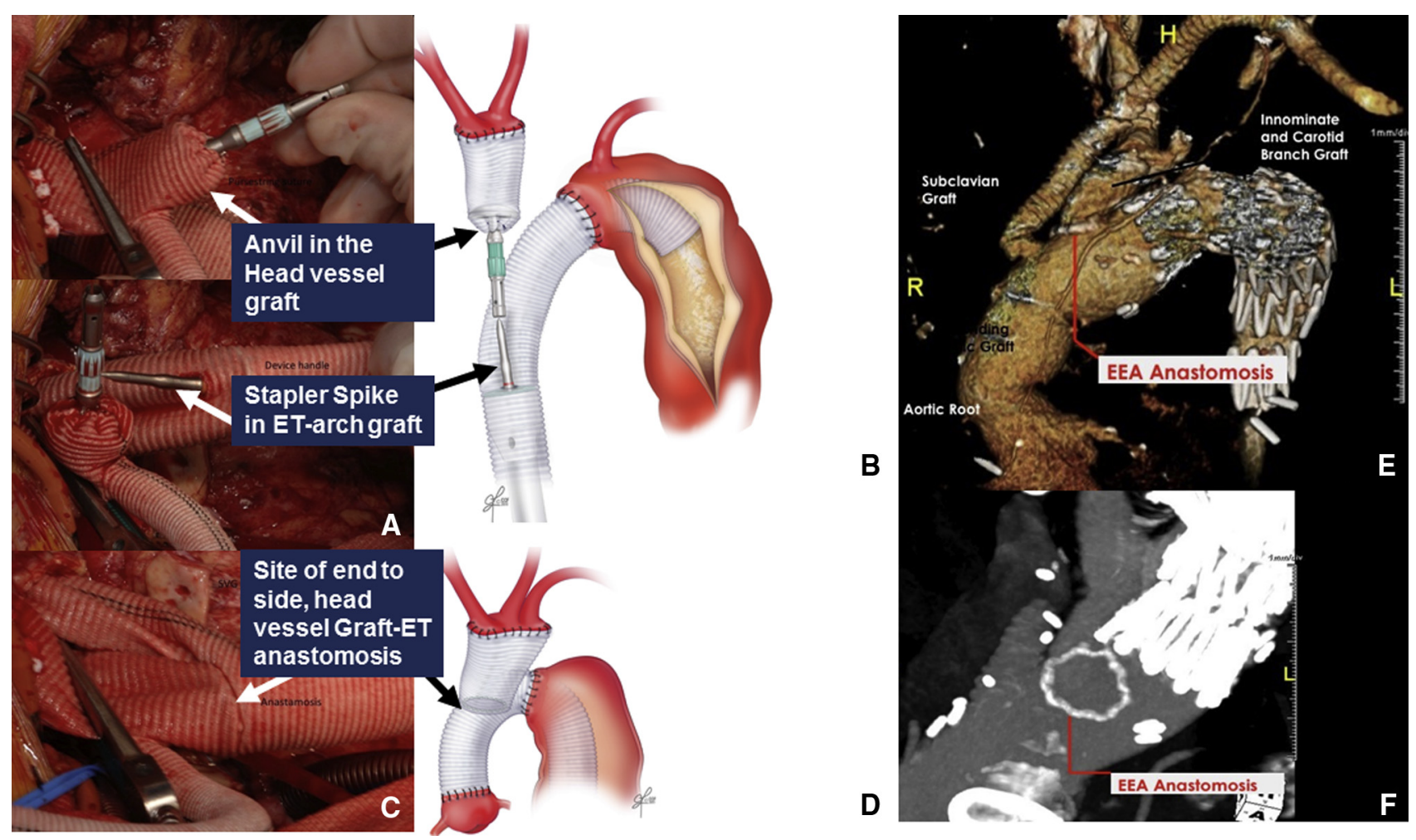

FIGURE 2. Intraoperative images (A and C) and artist illustrations (B and D) showing the use of end-to-end anastomotic (EEA) stapler for prosthetic graftto-prosthetic graft anastomosis and postoperative computed tomography showing stable anastomosis (E and $\mathrm{F}$ ). Illustrations show the anvil inside the elephant trunk (ET) graft that is brought out through the opening to lock in position with the trocar in the head vessel graft. An end-to-side anastomosis is then performed.

quicker medium vessel anastomoses during hybrid thoracoabdominal repair. Despite these limited experiences, the vast majority of vascular anastomoses are still performed by direct suturing. The large size of the EEA staples limits their use to grafts larger than $21 \mathrm{~mm}$ in diameter; however, the technique can be used for both end-to-side and end-to end-graft anastomoses.

Potential complications of using an automated stapler for vascular anastomosis can include the risks of blood leakage, thrombosis, and fracture of the staples at the anastomotic site. Although we did not observe these complications in our experience, caution is warranted for all patients, especially in those with atherosclerotic disease undergoing aortic arch reconstruction, who are at high risk for postoperative stroke. The 1 patient in our series who had stroke had a history of stroke caused by embolization from the pseudoaneurysm, with residual weakness. Postrepair magnetic resonance angiography showed bilateral infarcts in the vertebrobasilar distribution, not the anterior circulation. This was not due to staple embolization, and in all follow-up imaging the metallic anastomoses have remained intact. Patients undergoing complex aortic surgery have extensive disease and often require subsequent reoperations for progressive disease; imaging surveillance should therefore be performed routinely. ${ }^{13,14}$
In this series, all automated EEA stapler anastomoses were performed between prosthetic surgical grafts in an end-to-end or end-to-side fashion. Theoretically, an automated anastomosis could also be performed between a

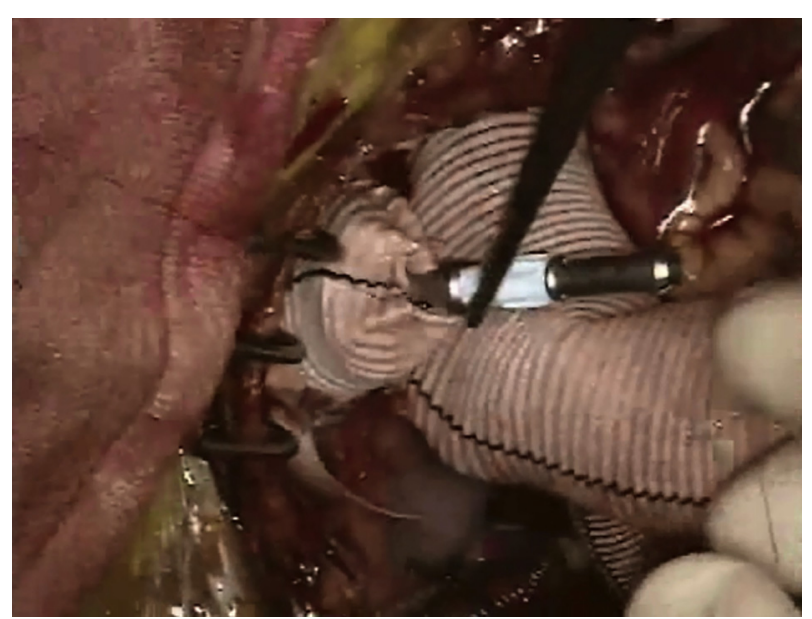

VIDEO 1. Short intraoperative video clip demonstrates the application of the stapler to perform an end-to-side graft-to-graft anastamosis. Video available at http://www.jtcvsonline.org/article/S0022-5223(16)30669-9/ addons. 


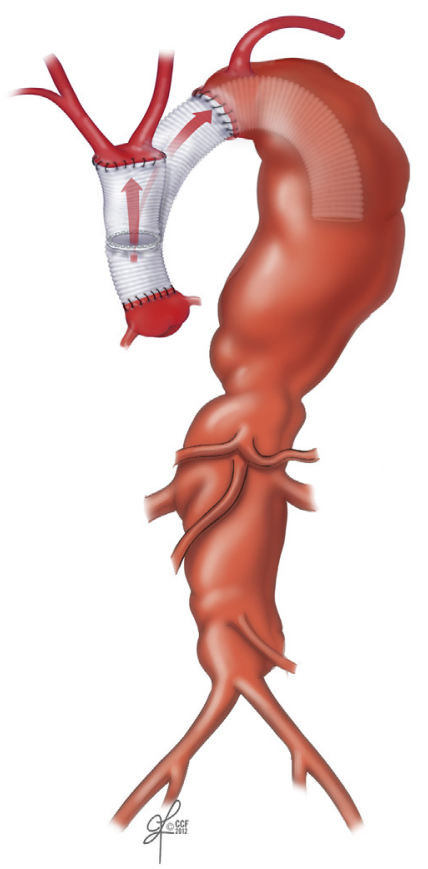

A

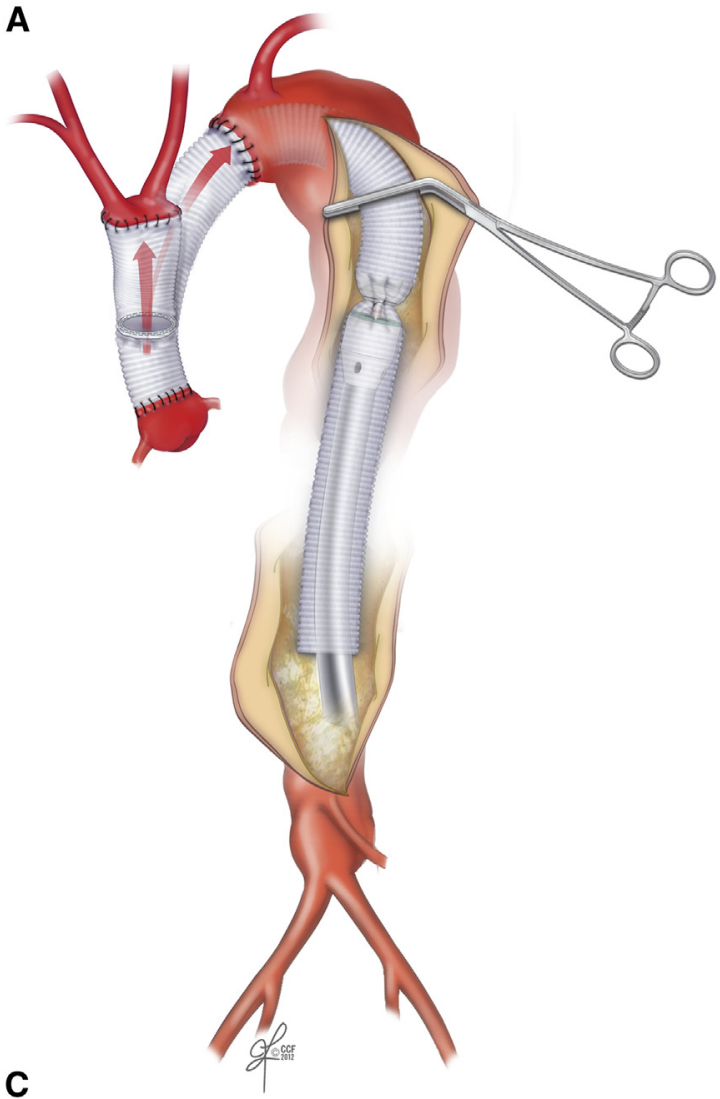

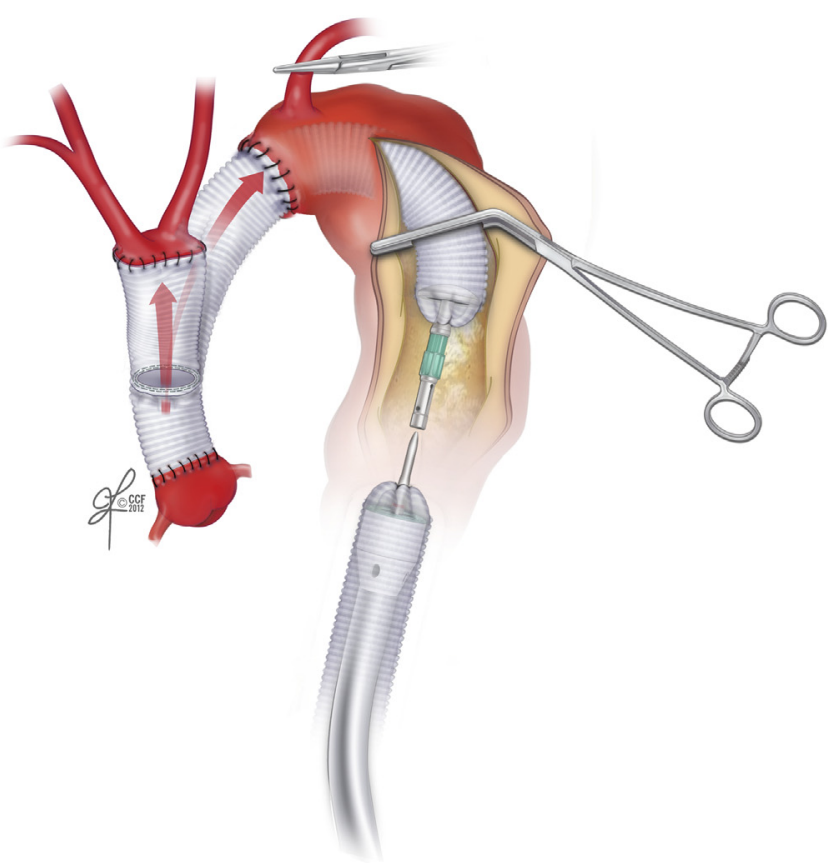

B

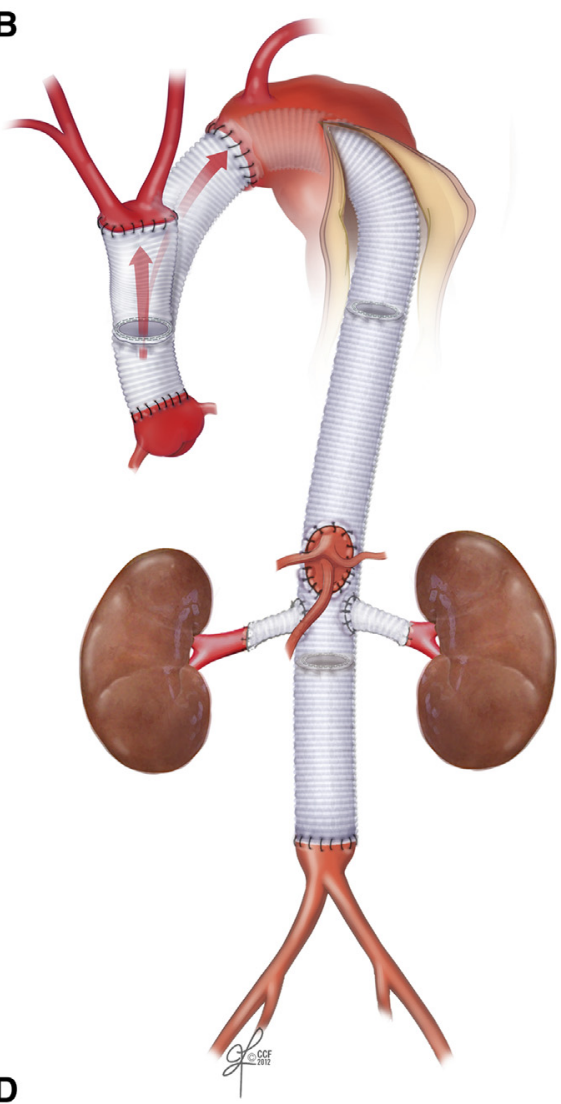

FIGURE 3. Demonstration of the use of end-to-end anastomotic stapler in performing the prosthetic graft-to-prosthetic graft, end-to-end anastomosis between the graft from the first-stage elephant trunk (A) and the completion thoracoabdominal graft (D). After clamping the elephant trunk, the anvil and stapler are quickly secured with purse strings to the 2 ends of the grafts (B). Then the ends of the stapler device are mated and deployed to complete the anastamosis (C). 


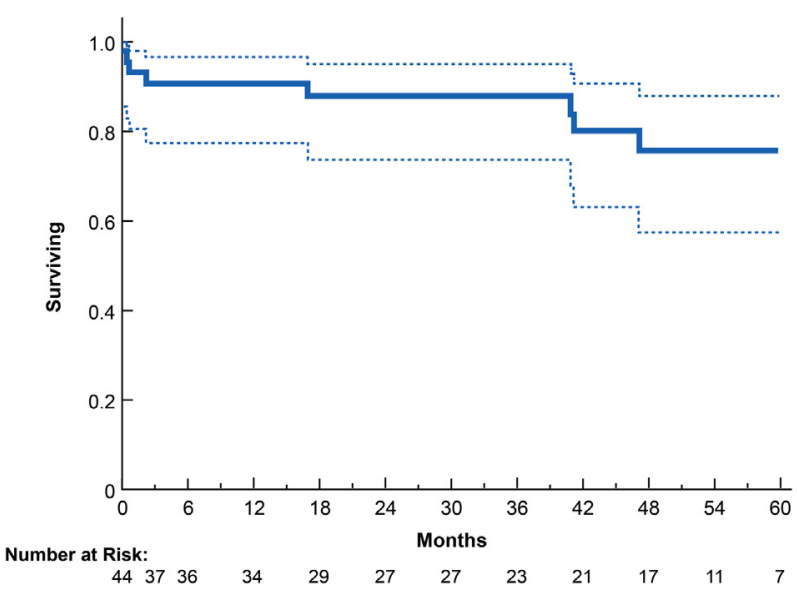

FIGURE 4. Long term survival.

surgical graft and the native aortic tissue. The current experimental data for that type of analysis are limited. We intentionally did not use this approach in our experience. A potential concern is the risk of dehiscence or leakage from the anastomotic site that may be caused by highpressure blood flow and remodeling of native tissue. Preliminary experimental data suggest that automated anastomosis with a stapler between graft and native aorta can withstand high-pressure circulation. Takata and colleagues ${ }^{11}$ compared the strength of the anastomosis of a surgical graft to porcine aorta between a surgically sutured anastomosis and that performed with an EEA stapler. The intraluminal pressure from blood flow was steadily increased. They reported that spurting leakage from anastomotic site did not occur until pressures of $332 \pm 84 \mathrm{~mm} \mathrm{Hg}$ were reached in the manual suture group, versus $440 \pm 98 \mathrm{~mm} \mathrm{Hg}$ in the automated stapler group $(P=.019)$, suggesting that the anastomosis resulting from use of an automated EEA stapler is resistant to high pressures similar to manual suturing. Further studies are needed to assess the durability of this approach in a chronic animal model.

The current commercially available EEA stapler devices are not specifically designed to perform vascular end-to-end anastomosis and come in a limited selection of sizes. In addition, in their current iteration, these devices create an inverted anastomosis that could potentially be flow limiting if applied to smaller vasculature. In our experience, EEA stapler use was limited to performing very large graft-tograft anastomoses only. The use of automated EEA stapling could be expanded by developing new devices that offer a variety of sizes and an everted type of anastomosis. These improvements could increase the applicability and use of automated staplers for performing anastomoses in multiple vascular beds consisting of medium and large vessels. Furthermore, development of such devices offers the potential to facilitate the development of less-invasive surgical techniques.
In summary, our early experience with the use of an endoluminal stapler for prosthetic graft-to-prosthetic graft anastomoses has demonstrated the safety and durability of this technique in performing a hemostatic anastomosis. According to reliable imaging follow-up, there were no complications specific to the device, and the integrity of the anastomosis has remained intact. Further evaluation and refinement of this technique are warranted.

\section{Conflict of Interest Statement}

Authors have nothing to disclose with regard to commercial support.

\section{References}

1. Khaladj N, Shrestha M, Meck S, Peterss S, Kamiya H, Kallenbach K, et al. Hypothermic circulatory arrest with selective antegrade cerebral perfusion in ascending aortic and aortic arch surgery: a risk factor analysis for adverse outcome in 501 patients. J Thorac Cardiovasc Surg. 2008;135:908-14

2. Svensson LG, Rushing GD, Valenzuela ES, Rafael AE, Batizy LH, Blackstone EH, et al. Modifications, classification, and outcomes of elephanttrunk procedures. Ann Thorac Surg. 2013;96:548-58.

3. Roselli EE, Subramanian S, Sun Z, Idrees J, Nowicki E, Blackstone EH, et al. Endovascular versus open elephant trunk completion for extensive aortic disease. J Thorac Cardiovasc Surg. 2013;146:1408-16; discussion 1416-7.

4. Roselli EE, Sepulveda E, Pujara AC, Idrees J, Nowicki E. Distal landing zone open fenestration facilitates endovascular elephant trunk completion and false lumen thrombosis. Ann Thorac Surg. 2011;92:2078-84.

5. Xu QR, Wang KN, Wang WP, Zhang K, Chen LQ. Linear stapled esophagogastrostomy is more effective than hand-sewn or circular stapler in prevention of anastomotic stricture: a comparative clinical study. J Gastrointest Surg. 2011; 15:915-21.

6. Korolija D. The current evidence on stapled versus hand-sewn anastomoses in the digestive tract. Minim Invasive Ther Allied Technol. 2008;17:151-4.

7. Markar SR, Karthikesalingam A, Vyas S, Hashemi M, Winslet M. Hand-sewn versus stapled oesophago-gastric anastomosis: systematic review and meta-analysis. J Gastrointest Surg. 2011;15:876-84.

8. Gummert JF, Opfermann U, Jacobs S, Walther T, Kempfert J, Mohr FW, et al. Anastomotic devices for coronary artery bypass grafting: technological options and potential pitfalls. Comput Biol Med. 2007;37:1384-93.

9. Sabik JF, Nemeh H, Lytle BW, Blackstone EH, Gillinov AM, Rajeswaran J, et al. Cannulation of the axillary artery with a side graft reduces morbidity. Ann Thorac Surg. 2004; 77:1315-20.

10. Shifrin EG, Moore WS, Bell PR, Kolvenbach R, Daniline EI. Intravascular stapler for "open" aortic surgery: preliminary results. Eur J Vasc Endovasc Surg. 2007;33:408-11.

11. Takata M, Watanabe G, Ohtake H, Ushijima T, Yamaguchi S, Kikuchi Y, et al Automatic aortic anastomosis with an innovative computer-controlled circular stapler for surgical treatment of aortic aneurysm. J Thorac Cardiovasc Surg. 2011;141:1265-9.

12. Lachat M, Mayer D, Criado FJ, Pfammatter T, Rancic Z, Genoni M, et al. New technique to facilitate renal revascularization with use of telescoping selfexpanding stent grafts: VORTEC. Vascular. 2008;16:69-72.

13. Idrees JJ, Roselli EE, Wojnarski CM, Feng K, Aftab M, Johnston DR, et al. Prophylactic stage 1 elephant trunk for moderately dilated descending aorta in patients with predominantly proximal disease. J Thorac Cardiovasc Surg. 2015; 150:1150-5.

14. Roselli EE, Idrees JJ, Lowry AM, Masabni K, Soltesz EG, Johnston DR, et al Beyond the aortic root: staged open and endovascular repair of arch and descending aorta in patients with connective tissue disorders. Ann Thorac Surg. 2016; 101:906-12.

Key Words: aneurysm, aortic surgery, circular stapler, elephant trunk, end-to-end anastomosis, thoracoabdominal 